\title{
Evaluación Postural y Prevalencia de Hipercifosis e Hiperlordosis en Estudiantes de Enseñanza Básica
}

\author{
Postural Evaluation and Prevalence of Hyperkyphosis and \\ Hyperlordosis in Students of Elementary School
}

\author{
Leonidas Brito-Hernández ${ }^{1,4}$; Omar Espinoza-Navarro'; Jorge Díaz Gamboa² \& Pablo A. Lizana ${ }^{3}$
}

BRITO-HERNÁNDEZ, L.; ESPINOZA-NAVARRO, O.; DÍAZ, G. J. \& LIZANA, P. A. Evaluación postural y prevalencia de hipercifosis e hiperlordosis en estudiantes de enseñanza básica. Int. J. Morphol., 36(1):290-296, 2018.

RESUMEN: El alineamiento incorrecto de la columna vertebral origina estrés y una tensión innecesaria que afecta el aparato locomotor. El objetivo de este trabajo fue identificar y establecer la prevalencia de hipercifosis e hiperlordosis y su relación con el índice de masa corporal (IMC) e índice cintura-talla (ICT) en estudiantes de 12 años de edad en Arica, Chile. Ochenta estudiantes de sexo masculino $(\mathrm{n}=80)$ de tres tipos de subvención escolar fueron reclutados. Se les evaluó peso $(\mathrm{kg})$, talla $(\mathrm{m})$, IMC e ICT como indicador de riesgo metabólico. Para la evaluación postural de hipercifosis e hiperlordosis se utilizó el test de flechas sagitales. Para los análisis estadísticos fueron ocupadas las pruebas Kruskal-Wallis, ANOVA de una vía y exacta de Fisher y Chi-cuadrado para las variables categóricas. Los resultados muestran una prevalencia total de alteración postural de un 57,5 \%, un IMC de sobrepeso/obesidad de un 52,5 $\%$ y un $35 \%$ de riesgo metabólico. No se observó una asociación entre IMC, ICT y alteraciones de columna, excepto en ICT e hipercifosis. Las alteraciones más frecuentes fueron hiperlordosis seguida de hipercifosis con un 38,8 \% y 25,7 \% respectivamente, donde las mayores prevalencias se registraron en el establecimiento municipal. En la muestra estudiada se reportan altas prevalencias de hipercifosis e hiperlordosis que no presentan asociación con los índices de sobrepeso y obesidad. Además, las mayores prevalencias de alteraciones posturales en el plano sagital se reportaron en el establecimiento municipal. La prevención de alteraciones posturales y el control del sobrepeso y obesidad deben realizarse precozmente en todo el sistema educativo.

PALABRAS CLAVE: Columna vertebral; Obesidad; Escolares; IMC; Curva Cifótica.

\section{INTRODUCCIÓN}

El mantenimiento y el control de la postura en diferentes posiciones aseguran la integridad y la integración de los diferentes sistemas del cuerpo humano, asegurando un efectivo y eficiente funcionamiento corporal como un todo. Los estudios coinciden en señalar que la postura está influenciada por tres factores fundamentales: la herencia, la enfermedad y el hábito (Santonja et al, 2006; Lafond et al., 2007; Zurita et al., 2008).

Las alteraciones posturales conllevan a un alineamiento incorrecto que origina estrés y una tensión innecesaria que afecta a los huesos, articulaciones, ligamentos y músculos (del Sol \& Hunter, 2004; Espinoza-Navarro et al., 2009; Yamamoto et al., 2015).
Durante el período de crecimiento se provoca en los niños desequilibrios en el aparato locomotor, circunstancia que supone un serio peligro para el escolar, incrementando la adopción de actitudes posturales inadecuadas con el riesgo de aparición de deformidades espinales que, con el transcurso del tiempo, pueden desencadenar en limitaciones funcionales (Viero Badaró et al., 2015).

Las alteraciones de la postura corporal, además de un considerable defecto estético, disminuyen su capacidad de trabajo, desalineaciones del aparato locomotor son frecuentes y la mayoría de ellas son asintomáticas, por lo que los escolares que las presentan no suelen conocer de su existencia (Santonja et al.). 
La cifosis es una curvatura sagital de la columna en la que el vértice de la curva tiene una dirección posterior. La cifosis de $40^{\circ}$ a $49^{\circ}$ está en el límite de la normalidad y las curvaturas mayores de $50^{\circ}$ se consideran como hipercifosis, que suele aparecer entre los 12-16 años, con predominio en sexo masculino, afecta fundamentalmente a la región posterior, en ocasiones puede afectar a la región lumbar y entonces el dolor es más evidente (Zurita et al., 2008, 2014). La lordosis es una flexión de la columna hacia posterior, que puede corresponder a la postura normal en la columna cervical y lumbar para compensar la cifosis torácica, pero que también puede tratarse de una situación anormal cuando es muy pronunciada. Estudios realizados por Yuing et al. (2010) determinan que un ángulo lordótico inferior a $\operatorname{los} 23^{\circ}$ es una hipolordosis y ángulos superiores a $\operatorname{los} 68^{\circ}$ se denomina hiperlordosis.

Para determinar la existencia de estas patologías, uno de los procedimientos más utilizados es el test de "flechas sagitales" que es un método de mediciones lineales, aceptado por su disponibilidad, sencillez, fácil reproductibilidad y validez (Rillardon et al., 2003; Santonja et al.; Yuing et al.).

Por otro lado, se ha reportado una alta prevalencia de sobrepeso y obesidad en niños y adolescentes en Chile (Lizana et al., 2011, 2016, 2017; Lizana Arce et al., 2012), que influiría en los efectos de las malas posturas y la presencia de patologías cardiovasculares, que aumentan en forma directa con la edad (MINSAL, 2004; OMS, 2017). La obesidad, según Crocker \& Yanovski (2009), es una enfermedad crónica de origen multifactorial, producida por un balance energético positivo mantenido en el tiempo, traduciéndose posteriormente en una acumulación excesiva de peso y masa grasa corporal, lo cual es perjudicial para la salud. El exceso de grasa en los niños se relaciona con riesgo cardiovascular y diabetes tipo 2 en la edad adulta, un buen método antropométrico para medir la adiposidad central es la relación cintura-estatura, cuando esta relación es $\geq 0,50$, identifica a población de riesgo (Arnaiz et al., 2014). Ashwell \& Gibson (2016) determinan que el índice cintura-talla (ICT) es mejor predictor que el índice de masa corporal (IMC) como indicador de riesgo de salud temprana. Factores intervinientes sobre la postura corporal de la población han sido motivo de creciente preocupación (Ainhagne \& Santhiago, 2009).

La presente investigación tiene como objetivo establecer la prevalencia de las alteraciones posturales hipercifosis e hiperlordosis y su relación con el IMC e ICT según tipo de subvención en escolares de enseñanza básica de Arica, Chile.

\section{MATERIAL Y MÉTODO}

Este estudio corresponde a un diseño del tipo transversal. Ochenta estudiantes de sexo masculino de 12 años de edad de tres diferentes tipos de subvención escolar (particular, subvencionado y municipal) de la ciudad de Arica aceptaron participar. Los criterios de inclusión fueron: estudiantes de sexo masculino, estudiantes con 12 años de edad cumplidos. Sujetos con evidencia de anormalidades espinales congénitas fueron excluidos del estudio, también se excluyeron a escolares de sexo femenino debido a que se evalúa con torso descubierto. Luego de firmar los asentimientos y consentimiento informado que fueron aprobadas por el Comité Ético Científico de la Universidad de Tarapacá, Arica, Chile, los participantes fueron evaluados entre los meses de agosto y noviembre del año 2016 según: peso, talla, perímetro de cintura, IMC e ICT. Los instrumentos de medición fueron: plomada Rottweiler (Evol 3730, China); regla técnica milimetrada precisión $1 \mathrm{~mm}$ (Faber Castell, Stein, Germany); balanza con tallímetro portátil precisión de 100 g y 1 mm (ADE M318300, West Germany); cinta antropométrica precisión de 0,1 cm (Lufkin W606 pm CM, Texas, USA) y cámara fotográfica (Samsung Smart WB800F, Daegu, Corea del Sur).

Para calcular el IMC, se aplicó formula de Quetelet (peso en kilogramos/talla en metros al cuadrado) y se clasificó a los estudiantes en bajopeso, normopeso, sobrepeso y obesidad, según edad y sexo (MINSAL). Para calcular el ICT, se utilizó punto de corte 0,50 según Ashwell \& Gibson. Para evaluación postural de hipercifosis e hiperlordosis se utilizó el test de flechas sagitales, mediante la determinación de la distancia entre la vertical de la plomada hasta el punto más alejado de la misma. (Santonja et al.; Zurita et al., 2008; Yuing et al.).

Los datos fueron analizados con el programa estadístico STATA 12.0. La normalidad de la distribución de los datos fue evaluada usando la prueba de Shapiro-Wilk. Para evaluar las diferencias entre los grupos se ocupó la prueba Kruskal-Wallis y ANOVA de un factor. Para evaluar las asociaciones fueron utilizadas las pruebas de chi-cuadrado y exacta de Fisher. Los niveles de confianza fueron del $95 \%$, con un valor alfa $\mathrm{p}<0,05$.

\section{RESULTADOS}

En la Tabla I se observan las características antropométricas de los estudiantes de los diferentes colegios en estudio con un peso promedio de 52,9 $\pm 12,2$; talla 
Tabla I. Características generales de los escolares de enseñanza básica segundo ciclo según tipo de establecimiento (n=80). Arica, Chile. Agosto - Noviembre, 2016.

\begin{tabular}{|c|c|c|c|c|c|}
\hline & Total & $\begin{array}{l}\text { Privado } \\
(\mathrm{n}=26)\end{array}$ & $\begin{array}{l}\text { Subvencionado } \\
\quad(\mathrm{n}=27)\end{array}$ & $\begin{array}{l}\text { Municipal } \\
(\mathrm{n}=27)\end{array}$ & $\mathrm{p}$-valor \\
\hline Peso (kg) & $52,9 \pm 12,2$ & $53,7 \pm 10,0$ & $54,8 \pm 12,7$ & $51,0 \pm 13,6$ & $0,372^{\mathrm{a}}$ \\
\hline Talla (m) & $1,54 \pm 0,1$ & $1,55 \pm 0,1$ & $1,57 \pm 0,1$ & $1,52 \pm 0,1$ & $0,070^{\mathrm{b}}$ \\
\hline $\operatorname{IMC}\left(\mathrm{kg} / \mathrm{m}^{2}\right)$ & $22 \pm 3,8$ & $22,2 \pm 2,9$ & $22,1 \pm 3,8$ & $21,9 \pm 4,4$ & $0,954^{\mathrm{b}}$ \\
\hline Bajo peso & $1,1(1)$ & $0(0)$ & $0(0)$ & $3,7(1)$ & $0,889^{\mathrm{c}}$ \\
\hline Normopeso \% (n) & $46,3(37)$ & $38,5(10)$ & $48,2(13)$ & $51,9(14)$ & \\
\hline Sobrepeso \% (n) & $22,5(18)$ & $26,9(7)$ & $22,2(6)$ & $18,5(5)$ & \\
\hline Obesidad \% (n) & $30,0(24)$ & $34,6(9)$ & $29,6(8)$ & $25,9(7)$ & \\
\hline Bajo peso/Normopeso \% (n) & $47,5(38)$ & $38,5(10)$ & $48,2(13)$ & $55,6(15)$ & $0,500^{\mathrm{d}}$ \\
\hline Sobrepeso/Obesidad \% (n) & $52,5(42)$ & $61,5(16)$ & $51,9(14)$ & $44,4(12)$ & \\
\hline ICT & $47,4 \pm 6,0$ & $47,3 \pm 4,5$ & $47,0 \pm 5,8$ & $47,8 \pm 7,6$ & $0,918^{\mathrm{a}}$ \\
\hline Normal \% (n) & $65,0(52)$ & $57,7(15)$ & 70,4 (19) & $66,7(18)$ & $0,611^{\mathrm{d}}$ \\
\hline Riesgo metabólico \% (n) & $35,0(28)$ & $42,3(11)$ & $29,6(8)$ & $43,3(9)$ & \\
\hline
\end{tabular}

IMC, índice de masa corporal; ICT, índice cintura-talla. a Prueba Kruskal-Wallis. b Prueba ANOVA de un factor. c Prueba exacta de Fisher. d Prueba chi-cuadrado.

de $1,54 \pm 0,1$ y un IMC de $22 \pm 3,8$. Se observaron niveles de sobrepeso y obesidad de un $52,5 \%$, sin diferencias significativas entre los colegios. El ICT fue de 47,5 $\pm 6,0$ y el riesgo metabólico de la muestra fue de $35,0 \%$.

Las mayores prevalencias se reportan para hiperlordosis con un $38,8 \%$ y para hipercifosis con un 25,7 $\%$. Al comparar las alteraciones posturales entre los colegios, se observó en los estudiantes del colegio municipalizado las mayores prevalencias con un $44,4 \%$ para hiperlordosis y 33,3\% para hipercifosis en relación a los estudiantes del colegio particular y subvencionado (Tabla II).

El 57,5\% de la muestra de estudiantes presenta alguna alteración de columna en el plano sagital, que puede ser hipercifosis, hiperlordosis o ambas. Los estudiantes del establecimiento subvencionado son los que presentan mayores prevalencias de hipercifosis o hiperlordosis con un 63 $\%$ seguido del establecimiento municipal. Sin embargo, la mayor prevalencia de la condición combinada de hipercifosis e hiperlordosis se observa en el establecimiento municipal con el $14,8 \%$ (Tabla III).

Tabla II. Prevalencias de alteraciones posturales de columna vertebral en el plano sagital por tipo de establecimiento educacional. Arica, Chile. Agosto - Noviembre, 2016.

\begin{tabular}{lccccc}
\hline & Total & Privado & Subvencionado & Municipal & \multirow{2}{*}{ p-valor } \\
\cline { 3 - 4 } & & $(\mathrm{n}=26)$ & $(\mathrm{n}=27)$ & $(\mathrm{n}=27)$ & \\
\hline Curva lumbar & & & & & \\
$\quad$ Con Hiperlordosis & $38,8(31)$ & $30,7(8)$ & $40,7(11)$ & $44,4(12)$ & $0,574 \mathrm{a}$ \\
$\quad$ Sin Hiperlordosis & $61,2(49)$ & $69,3(18)$ & $59,3(16)$ & $55,6(15)$ & \\
Curva torácica & & & & & \\
$\quad$ Con Hipercifosis & $25,7(22)$ & $18,2(5)$ & $29,6(19)$ & $33,3(9)$ & $0,493^{\mathrm{a}}$ \\
$\quad$ Sin Hipercifosis & $72,5(58)$ & $80,8(21)$ & $70,4(8)$ & $66,7(18)$ & \\
\hline
\end{tabular}

Datos presentados en porcentaje y (frecuencia). a Prueba chi-cuadrado.

Tabla III. Prevalencias de hipercifosis o hiperlordosis y su prevalencia combinada por tipo de establecimiento educacional. Arica, Chile. Agosto-Noviembre, 2016.

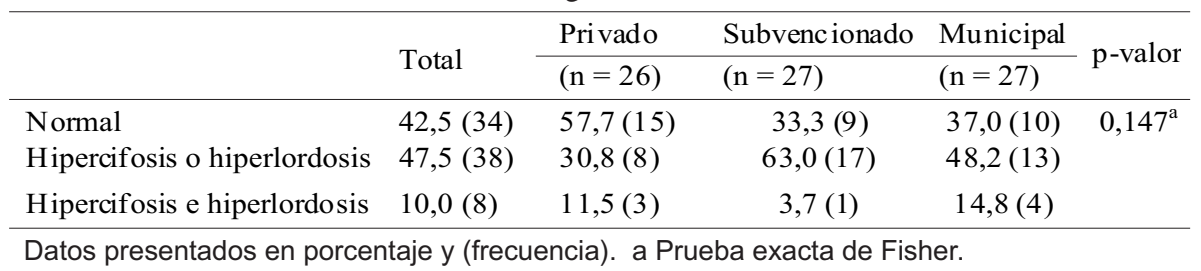


En la Tabla IV se reporta que la mayor prevalencia de hipercifosis se observa en los estudiantes normopeso, seguido de los obesos con un $22,7 \%$. Aspecto contrario en la hiperlordosis donde la mayor prevalencia se observa entre los escolares obesos con un 38,7 \%, seguido de un 32,3\% en la categoría normopeso. En el ICT existe una asociación significativa con hipercifosis, donde un 18,2\% de los escolares con riesgo metabólico presenta dicha condición. Por otro lado, el 38,7 \% de los sujetos que presenta riesgo metabólico también registra hiperlordosis.
Los resultados por estado nutricional mediante IMC indican que las mayores prevalencias de hipercifosis o hiperlordosis se observan en los estudiantes normopeso con $42,1 \%$ y para la prevalencia combinada de hiperlordosis e hiperlordosis con un $50 \%$ ( Tabla V). Resultados similares se reportan con el ICT, donde las mayores prevalencias se observan en la categoría normal. Sin embargo, el 39,5\% de los estudiantes con riesgo metabólico presentan hipercifosis o hiperlordosis.

Tabla IV. Prevalencias de hipercifosis y de hiperlordosis por categoría del índice de masa corporal e índice cintura talla. Arica, Chile. Agosto-Noviembre, 2016.

\begin{tabular}{lcccc}
\hline & Normal & Hipercifosis & Hiperlordosis & p-valor \\
\hline Estado nutricional (IMC) & & & & \\
Bajopeso & $1,7(1)$ & $0(0)$ & - & $0,290 \mathrm{a}$ \\
Normopeso & $39,7(23)$ & $63,6(14)$ & - & \\
Sobrepeso & $25,9(15)$ & $13,6(3)$ & - & \\
Obesidad & $32,8(19)$ & $22,7(5)$ & - & \\
Estado nutricional (IMC) & & & & \\
Bajopeso & $2,0(1)$ & - & $0(0)$ & $0,144 \mathrm{a}$ \\
Normopeso & $55,1(27)$ & - & $32,3(10)$ & \\
Sobrepeso & $18,4(9)$ & - & $29,0(9)$ & \\
Obesidad & $24,5(12)$ & - & $38,7(12)$ & \\
ICT & & & & $0,043 \mathrm{a}$ \\
Normal & $58,6(34)$ & $81,8(18)$ & - & \\
Riesgo metabólico & $41,4(24)$ & $18,2(4)$ & - & $0,580^{\mathrm{b}}$ \\
ICT & & & & \\
Normal & $67,4(33)$ & - & $61,3(19)$ & \\
Riesgo metabólico & $32,7(16)$ & - & $38,7(12)$ & \\
\hline
\end{tabular}

Abreviaturas: IMC, índice de masa corporal. ICT, índice cintura-talla. Datos presentados en porcentaje y (frecuencia). a Prueba exacta de Fisher. b Prueba chicuadrado.

Tabla V. Prevalencias de hipercifosis o hiperlordosis y su prevalencia combinada por categoría del índice de masa corporal e índice cintura talla. Arica, Chile. Agosto-Noviembre, 2016

\begin{tabular}{lcccc}
\hline & Normal & Hipercifosis o hiperlordosis & Hipercifosis e hiperlordosis & p-valor \\
\hline Estado nutricional (IMC) & & & \\
$\quad$ Bajopeso & $2,9(1)$ & $0(0)$ & $0(0)$ & $0,834 \mathrm{a}$ \\
$\quad$ Normopeso & $50(17)$ & $42,1(16)$ & $50,0(4)$ & \\
Sobrepeso & $23,5(8)$ & $21,1(8)$ & $25,0(2)$ & \\
Obesidad & $23,5(8)$ & $36,8(14)$ & $25,0(2)$ & \\
ICT & & & & \\
$\quad$ Normal & $64,7(22)$ & $60,5(23)$ & $12,5(7)$ & $0,409 \mathrm{a}$ \\
$\quad$ Riesgo metabólico & $35,3(12)$ & $39,5(15)$ & $10,5)$ & \\
\hline
\end{tabular}

IMC, índice de masa corporal. ICT, índice cintura-talla. Datos presentados en porcentaje y (frecuencia). a Prueba exacta de Fisher. 


\section{DISCUSIÓN}

Alineamientos incorrectos de la columna vertebral afectan a los huesos, articulaciones, ligamentos y músculos (Yamamoto et al.; Santonja et al.). La espalda al estar encorvada tiene más posibilidades de debilitar los músculos del abdomen y el diafragma, teniendo como consecuencia la aparición de un vientre abultado y problemas respiratorios. Del mismo modo, puede provocar dolor que con el transcurso del tiempo irá en aumento y es responsable de una imagen corporal deteriorada lo que conlleva niveles más bajos de autoestima (del Sol \& Hunter; Martelli \& Traebert, 2006).

Los resultados de esta investigación muestran una alta prevalencia de hipercifosis e hiperlordosis, con resultados similares a lo reportado Martínez Marínz \& Fonseca (2013). Otros estudios detectaron una prevalencia de un 71,3 $\%$ para hipercifosis y de un 28,8\% para hiperlordosis en estudiantes de Colombia (Castiblanco et al. 2013). Bueno $\&$ Rech (2013), reportan valores de $16 \%$ y 27,9 \% para hipercifosis e hiperlordosis respectivamente en población preadolescente de Brasil, resultados que son similares a los reportados en este trabajo, donde se observó mayores prevalencias de hiperlordosis que de hipercifosis.

La literatura muestra resultados dispares, lo que hace necesario analizar las diferentes metodologías ocupadas. El estudio realizado por Viero Badaró et al., determinaron grandes variaciones de prevalencia para alteraciones posturales, con valores de $15,3 \%$ a $78 \%$ para hiperlordosis y de $9,0 \%$ a un $53,8 \%$ para hipercifosis, afirmando que esto se debe a la gran variedad de sistema o instrumental utilizado y además influenciado por el sexo y el grupo etáreo. En este caso, la presente investigación reclutó sólo a estudiantes del sexo masculino. En el futuro se deberían hacer trabajos que también incluyan al sexo femenino.

Al relacionar prevalencia de hipercifosis e hiperlordosis con el IMC y el ICT, no se observó una asociación, además se observó que las mayores alteraciones de columna se encuentra en niños normopesos y de bajo riesgo metabólico. Similares resultados presentan Castiblanco et al., quienes determinaron en los jóvenes un sobrepeso del $40,22 \%$ pero no encontraron una relación con el riesgo cardiovascular, según índice de cintura. Sin embargo, sí se pudo reportar una asociación entre ICT e hipercifosis, aspecto que indica que se deben hacer mayores y más precisos estudios sobre métodos de evaluación de la adiposidad y su distribución, puesto que se ha observado que el IMC no es un buen indicador de adiposidad (Rothman, 2008; Lizana et al., 2016).
Los altos índices de sobrepeso y obesidad son una realidad latente en Latinoamérica (Rivera et al., 2014) y Chile no es la excepción (Lizana et al., 2016, 2017). Para la evaluación del sobrepeso y obesidad el IMC ha sido ampliamente utilizado, sin embargo a través de este índice no se reportan los componentes grasos ni su distribución (Rothman) como tampoco la masa muscular. Este aspecto podría ser importante en estudios posteriores puesto que se ha visto que tiene influencia en desordenes postulares en niños (Wyszyn'ska et al., 2016).

La prevalencia de estas anormalidades posturales y las prevalencias observadas por el tipo de subvención, donde menores porcentajes de alteraciones posturales se presentan en el colegio de tipo particular y las mayores prevalencias en el colegio de subvención municipal se podría relacionar a los diferentes ingresos que reciben las familias de colegios particulares. En este caso, las familias con mayor ingreso económico tienen mayor acceso a los sistemas de salud y atención con médicos especialistas (Espinoza-Navarro et al., 2013). Viero Badaró et al., hacen referencia a que los colegios de mejores ingresos presentan una estructura y muebles más adecuados en sus aulas. Es importante que este tipo de estudios sigan realizándose para permitir una mejor estandarización de las mediciones y el instrumental ocupado, como también incorporar variables de nivel socioeconómico para determinar su efecto en las prevalencias de alteraciones posturales. Los cambios en la curvatura de la columna vertebral, suelen preceder a los síntomas neurológicos, provocando en los niños dolor. La detección precoz permite la corrección oportuna con ejercicios terapeúticos (Olmedo-Buenrostro et al., 2006; Yuing et al.).

Limitaciones. Una de las limitaciones es la naturaleza del estudio (transversal) que no permite un seguimiento de los sujetos. Además, en este estudio la evaluación de la composición corporal se vio limitada a IMC e ICT, las cuales no permiten la evaluación de los componentes grasos y musculares. Esto sería relevante estudiarlo en próximos estudios puesto que existen antecedentes que indican que tendrían relación con desordenes posturales en niños (WyszyNska et al.,). Por otro lado, se ha observado que el nivel socioeconómico tiene una asociación con la adiposidad en escolares (Lizana et al., 2017). Sería importante evaluar si este tipo de variable influye en los desórdenes posturales. Por último, en este estudio se evaluó a estudiantes de sexo masculino, próximos estudios deberían incorporar a ambos sexos.

En resumen, se concluye que la muestra en estudio presenta altas prevalencias de hipercifosis e hiperlordosis, que no presentan relación con el sobrepeso y obesidad ni 
con riesgo metabólico, excepto en ICT e hipercifosis. Se observa que los estudiantes de establecimiento particular presentan menores las prevalencias de sobrepeso y de alteraciones posturales. Fuertes estudios se deben realizar para normalizar y estandarizar los sistemas e instrumental de medición de alteraciones posturales, además de implementar políticas públicas adecuadas para enfrentar tanto la problemática de las alteraciones posturales como el incremento del sobrepeso y obesidad.

AGRADECIMIENTOS. Este trabajo fue subvencionado por el proyecto UTA Mayor $\mathrm{N}^{\circ}$ 5750-15, Universidad de Tarapacá, Arica-Chile y proyecto Tesis de Magíster UTA 2017 N $5755-17$. También agradecemos el constante apoyo de la Vicerrectoría de Investigación y Estudios Avanzados de la Pontificia Universidad Católica de Valparaíso, Chile. Las instituciones no tuvieron ningún papel en la recopilación de datos, análisis o redacción del presente manuscrito.

BRITO-HERNÁNDEZ, L.;ESPINOZA-NAVARRO, O.; DÍAZ, G. J. \& LIZANA, P. A. Postural evaluation and prevalence of hyperkyphosis and hyperlordosis in students of elementary school. Int. J. Morphol., 36(1):290-296, 2018.

SUMMARY: Incorrect alignment of the spinal column causes stress and unnecessary stress affecting the locomotor system. The objective of this study was to identify and establish the prevalence of hyperkyphosis and hyperlordosis and its relationship with body mass index (BMI) and waist-height ratio (WHR) in 12year-old students from Arica, Chile. Eighty male students $(n=80)$ from three types of school: public, private subsidized, and private nonsubsidized schools were recruited. They were assessed weight $(\mathrm{kg})$, height $(\mathrm{m}), \mathrm{BMI}$ and $\mathrm{WHR}$ as an indicator of metabolic risk. The sagittal arrow test was used for postural evaluation of hyperkyphosis and hyperlordosis. Kruskal-Wallis tests, one-way ANOVA and Fisher's exact test and Chi-square test were used for the categorical variables. The results show a total prevalence of postural alteration of $57.5 \%$, a BMI of overweight / obesity of $52.5 \%$ and a $35 \%$ of metabolic risk. There was no association between BMI, WHR and spinal alterations, except for WHR and hyperkyphosis. The most frequent alterations were hyperlordosis followed by hyperkyphosis with $38.8 \%$ and $25.7 \%$ respectively, where the highest prevalences were recorded in the public school. In the sample studied we report the high prevalence of hyperkyphosis and hyperlordosis that there were not association with the indexes of overweight and obesity. In addition, the higher prevalences of postural alterations in the sagittal plane were reported in the public school. The prevention of postural alterations and the control of overweight and obesity should be carried out early in the whole elementary education system.

KEY WORDS: Spinal Column; Obesity; School Children; BMI; Kyphosis Curve.

\section{REFERENCIAS BIBLIOGRÁFICAS}

Ainhagne, M. \& Santhiago, V. Cadeira e mochila escolares no processo de desenvolvimento da má postura e possíveis deformidades em crianças de 8-11 anos. Colloq. Vit., 1(1):1-7, 2009.

Arnaiz, P.; Grob, F.; Cavada, G.; Domínguez, A.; Bancalari, R.; Cerda, V.; Zamorano, J.; Fernández, M. \& García, H. Waist-to-height ratio does not change with gender, age and pubertal stage in elementary school children. Rev. Med. Chile, 142(5):574-8, 2014.

Ashwell, M. \& Gibson, S. Waist-to-height ratio as an indicator of 'early health risk': simpler and more predictive than using a 'matrix' based on BMI and waist circumference. BMJ Open, 6(3):e010159, 2016.

Bueno, R. C. S. \& Rech, R. R. Postural deviations of students in Southern Brazil. Rev. Paul. Pediatr., 31(2):237-42, 2013.

Castiblanco Díaz, J. R.; Silva, E. P.; Acosta, L. V. \& Campos, A. Caracterización postural en los preadolescentes del colegio Santo Tomás de Aquino en la ciudad de Bogotá. Mov. Cient., 7(1):105-13, 2013.

Crocker, M. K. \& Yanovski, J. A. Pediatric obesity: Etiology and treatment. Endocrinol. Metab. Clin. North Am., 38(3):525-48, 2009.

del Sol, M. \& Hunter, K. Postural evaluation of mapuche individuals in the coastal area of the IX Region in Chile. Int. J. Morphol., 22(4):339-42, 2004.

Espinoza-Navarro, O.; Olivares Urquieta, M.; Palacios Navarrete, P. \& Robles Flores, N. Prevalence of foot anomalies in schoolchildren between 6 and 12 years old of elementary education from Arica-Chile. Int. J. Morphol., 31(1):162-8, 2013.

Espinoza-Navarro, O.; Valle, S.; Berrios, G.; Horta, J.; Rodríguez, H. \& Rodríguez, M. Prevalence of postural alterations and effects of a program to improve the posture in children from Arica-Chile. Int. J. Morphol., 27(1): 25-30, 2009.

Lafond, D.; Descarreaux, M.; Normand, M. C. \& Harrison, D. E. Postural development in school children: a cross-sectional study. Chiropr. Osteopat., 15:1, 2007.

Lizana Arce, P.; Almagiâ Flores, A.; Simpson Lelievre, C.; Ivanovic Marincovic, D.; Binvignat Gutiérrez, O. \& Berral de la Rosa, F. Changes of somatotype in high school students, V region, Chile: 1985-2010. Nutr. Hosp., 27(1):270-5, 2012.

Lizana, A. P. A.; Almagia, F. A. A.; Simpson, L. M. C.; Binvignat, G. O.; Ivanovic, M. D. \& Berral de la Rosa, F. J. Approximation to the secular tendency of the nutritional state and body composition of high school students, V Region, Chile: 1985-2010. Int. J. Morphol., 29(2):473-8, 2011.

Lizana, P. A.; Gonzalez, S.; Lera, L. \& Leyton, B. Association between body composition, somatotype and socioeconomic status in Chilean children and adolescents at different school levels. J. Biosoc. Sci., 50(1):53-69, 2018.

Lizana, P. A.; Paula, C. V.; Araya, L.; Aguilera, F. \& Mora, M. Obesity, Body Fat Distribution, and Physical Activity in School-age Children: an Urban and Rural Comparison in Valparaíso, Chile. Biomed. Environ. Sci., 29(11):834-9, 2016.

Martelli, R. C. \& Traebert, J. Estudo descritivo das alterações posturais de coluna vertebral em escolares de 10 a 16 anos de idade: Tangará-SC, 2004. Rev. Bras. Epidemiol., 9(1):87-93, 2006.

Martínez Marínz, R. P. \& Fonseca, A. A. Deficiencias posturales en escolares de 8 a 12 años de una institución educativa pública, año 2010. Rev. Univ. Salud, 15(1):22-33, 2013.

Ministerio de Salud de Chile (MINSAL). Norma técnica de evaluación nutricional del niño de 6 a 18 años. Año 2003. Rev. Chil. Nutr., 31(2):128-37, 2004.

Olmedo-Buenrostro, B. A.; Enrique-Tene, C.; Díaz-Giner, V.; TrujilloHernández, B. \& Millán-Guerrero, R. O. Evaluación de un marco de referencia postural como prueba diagnóstica de postura lordótica lumbar. Gac. Med. Mex., 142(1):39-42, 2006.

Organización Mundial de la Salud (OMS). Obesidad y Sobrepeso. Gine- 
BRITO-HERNÁNDEZ, L.; ESPINOZA-NAVARRO, O.; DÍAZ, G. J. \& LIZANA, P. A. Evaluación postural y prevalencia de hipercifosis e hiperlordosis en estudiantes de enseñanza básica. Int. J. Morphol., 36(1):290-296, 2018

bra, Organización Mundial de la Salud (OMS), 2017. Disponible en: http://www.who.int/mediacentre/factsheets/fs311/es/

Rillardon, L.; Levassor, N.; Guigui, P.; Wodecki, P.; Cardinne, L.; Templier, A. \& Skalli, W. Validation of a tool to measure pelvic and spinal parameters of sagittal balance. Rev. Chir. Orthop. Reparatrice. Appar. Mot., 89(3):218-27, 2003.

Rivera, J. Á.; de Cossío, T. G.; Pedraza, L. S.; Aburto, T. C.; Sánchez, T. G. \& Martorell, R. Childhood and adolescent overweight and obesity in Latin America: a systematic review. Lancet Diabetes Endocrinol., 2(4):321-32, 2014.

Rothman, K. J. BMI-related errors in the measurement of obesity. Int. J. Obes. (Lond.), 32 Suppl. 3:S56-9, 2008.

Santonja, F.; Andújar, P. \& Ortín, E. Escoliosis. En: Arribás Blanco, J. M.; Castelló Fortet, J. R.; Rodríguez Pata, N.; Santonja Medina, F. \& Plazas, N. (Eds.). Cirugía Menor y Procedimientos en Medicina de Familia. Madrid, JARPYO, 2006.

Viero Badaró, A. F.; Ilha Nichele, L. F. \& Turra, P. Investigação da postura corporal de escolares em estudos brasileiros. Fisioter. Pesqui., 22(2):197-204, 2015.

Wyszyn`ska, J.; Podgórska-Bednarz, J.; Drza?-Grabiec, J.; Rachwa?, M.; Baran, J.; Czenczek-Lewandowska, E.; Leszczak, J. \& Mazur, A. Analysis of relationship between the body mass composition and physical activity with body posture in children. BioMed Res. Int., 2016:1851670, 2016.

Yamamoto, T.; Smith, C. E.; Suzuki, Y.; Kiyono, K.; Tanahashi, T.; Sakoda, S.; Morasso, P. \& Nomura, T. Universal and individual characteristics of postural sway during quiet standing in healthy young adults. Physiol. Rep., 3(3):e12329, 2015.

Yuing, F. T. A.; Almagia, A. F.; Lizana, P. J.; Rodríguez, R. F. J.; Ivanovic, D. M.; Binvignat, G. O.; Gallardo, L. R.; Nieto, C. F. \& Verdejo, S. A. Comparison of Two Method for Measuring the Lumbar Curve. Int. J. Morphol., 28(2):509-13, 2010.

Zurita Ortega, F.; Romero Cerezo, C.; Ruiz Rodríguez, L.; Martínez Martínez, A.; Fernández García, R. \& Fernández Sanchez, M. Influencia de las alteraciones raquídeas en la flexibilidad de los escolares. Rev. Int. Med. Cien. Act. Fis. Deporte, 8(32):282-98, 2008.

Zurita Ortega, F.; Ruiz Rodríguez, L.; Zaleta Morales, L.; Fernández Sánchez, M.; Fernández García, R. \& Linares Manrique, M. Análisis de la prevalencia de escoliosis y factores asociados en una población escolar mexicana mediante técnicas de cribado. Gac. Med. Mex., 150(5):432-9, 2014

\author{
Corresponding author: \\ Omar Espinoza-Navarro \\ Universidad de Tarapacá \\ Arica \\ CHILE
}

Email: omarespinoza091@gmail.com

Recibido : 13-08-2017

Aceptado: 22-01-2018 